Article

\title{
Comparison of Numerical Calculation Methods for Stem Diameter Retrieval Using Terrestrial Laser Data
}

\author{
Lei You ${ }^{1,2, *(\mathbb{D})}$, Jie Wei ${ }^{1}$, Xiaojun Liang ${ }^{3,4}$, Minghua Lou ${ }^{5}$, Yong Pang ${ }^{3,4}\left(\mathbb{D}\right.$ and Xinyu Song ${ }^{6,(\mathbb{D})}$ \\ 1 College of Computer and Information Technology, Xinyang Normal University, Xinyang 464000, China; \\ jiewei1999@foxmail.com \\ 2 Henan Key Lab of Analysis and Applications of Education Big Data, Xinyang Normal University, \\ Xinyang 464000, China \\ 3 Institute of Forest Resources Information Techniques, Chinese Academy of Forestry, Beijing 100091, China; \\ stanfordlxj@163.com (X.L.); pangy@ifrit.ac.cn (Y.P.) \\ 4 Key Laboratory of Forestry Remote Sensing and Information System, National Forestry and Grassland, \\ Beijing 100091, China \\ 5 Ningbo Academy of Agricultural Sciences, Ningbo 315040, China; mhlou1987@163.com \\ 6 College of Mathematics and Information Science, Xinyang Normal University, Xinyang 464000, China; \\ xysong88@163.com \\ * Correspondence: leiyou@xynu.edu.cn
}

Citation: You, L.; Wei, J.; Liang, X.;

Lou, M.; Pang, Y.; Song, X.

Comparison of Numerical

Calculation Methods for Stem

Diameter Retrieval Using Terrestrial

Laser Data. Remote Sens. 2021, 13

1780. https://doi.org/10.3390/

rs13091780

Academic Editor: Georgios Mallinis

Received: 19 March 2021

Accepted: 26 April 2021

Published: 2 May 2021

Publisher's Note: MDPI stays neutral with regard to jurisdictional claims in published maps and institutional affiliations.

Copyright: (c) 2021 by the authors. Licensee MDPI, Basel, Switzerland. This article is an open access article distributed under the terms and conditions of the Creative Commons Attribution (CC BY) license (https:// creativecommons.org/licenses/by/ $4.0 /)$

\begin{abstract}
Terrestrial laser scanning (TLS) can be used as a millimeter-level measurement tool for forest inventories. However, the stem diameter retrieval accuracy in sample plot scanning is not yet convincing. The errors in each step of stem diameter retrieval algorithms must be evaluated. In this study, six numerical calculation methods for the numerical calculation step, i.e., cylinder fitting (CYF), circle fitting (CF), convex hull line fitting (CLF), the proposed caliper simulation method (CSM), closure B-spline curve fitting (SP) and closure Bézier curve fitting with global convexity (SPC), were applied to stem diameter retrieval, and the similarities and differences were evaluated. The ovality, completeness and roughness were used to evaluate the stem slice point cloud quality. A total of 165 stem slice point clouds at breast height collected from three Larix kaempferi plots were used. Compared with the field-measured stem diameters at breast height (DBHs), the root mean square errors (RMSEs) of the CYF, CF, CLF, CSM, SP and SPC methods were $0.30 \mathrm{~cm}, 0.30 \mathrm{~cm}, 0.51 \mathrm{~cm}$, $0.51 \mathrm{~cm}, 0.56 \mathrm{~cm}$ and $0.54 \mathrm{~cm}$, respectively. Compared with the SPC method results, the RMSE of the CSM results was $0.05 \mathrm{~cm}$. The results illustrated that the CYF and CF methods performed the same, as did the CLF and CSM methods. Most DBHs retrieved by the CYF and CF methods were smaller than the field-measured DBHs, and most DBHs retrieved by the CLF, CSM, SP and SPC methods were larger than the field-measured DBHs. This study demonstrated that the CYF and CF methods perform the best and are the most robust, and the measurements by a diameter tape and a caliper are similar enough for forestry inventories. Evaluating and preprocessing stem slice point clouds is a potential way to improve stem diameter retrieval accuracy.
\end{abstract}

Keywords: TLS; stem diameter; stem diameter tape; caliper; cylinder fitting; circle fitting

\section{Introduction}

Terrestrial laser scanning (TLS) has emerged as an accurate means for nondestructively deriving three-dimensional (3D) forest structural attributes [1]. TLS has the capacity to obtain the 3D geometrical structure of a tree at the millimeter level, which is beyond the ability of traditional measurement tools [2]. After two decades of research, the tree attributes that can be automatically retrieved from TLS data include not only widely used forest inventories, such as tree location [3-5], stem diameter [6-10], stem height [9,11], stem crown width [9], but also those that cannot be directly measured using conventional tools, such as stem curve [12,13], stem volume [14,15] and other forestry parameters. However, TLS has not yet been considered an operational tool in forest inventories. In addition to the 
cost, there is another factor that is the difficulties in constructing automatic stem parameter retrieval methods that provide convincing measurement results [16].

Stem diameter is one of the most important parameters in forest inventories, as some important stem metrics, such as stem taper construction and merchantable stem volume [17], are calculated based on this parameter. Therefore, accurate retrieval of the stem diameter has become one of the important studies for forest inventories using TLS data.

There are many steps in a stem diameter retrieval algorithm, such as stem cross-section determination $[8,18]$, stem point selection [3,19], outliers removal $[20,21]$ and numerical computation [7]. The accuracy of retrieved stem diameter at breast height (DBH) using the circle fitting (CF) method is effected by the stem cross-section thickness, where the values obtained in the range of $1-10 \mathrm{~cm}$ are not significantly different and those obtained in the range of 10-100 $\mathrm{cm}$ are significantly different [19]. In practice, it is unrealistic to evaluate the errors introduced in all the steps of all stem diameter retrieval algorithms. However, all stem diameter retrieval algorithms can be divided into two major steps. The first is stem point preprocessing, which focuses on data collection and preprocessing, such as point cloud registration, ground point removal, stem location, stem cross-section determination and stem point selection. The next step is numerical calculation for stem diameter retrieval, which focuses on numerical computational methods, such as CF and cylinder fitting (CYF). The aims of the two steps are to provide stem points with high quality and to retrieve stem diameters with high precision. A satisfactory stem diameter is retrieved when both these aims are achieved. As a matter of fact, some studies have divided the stem diameter retrieval algorithm into two steps similar to the above. Such as, the first step is outliers removal $[20,21]$ or stem point selection $[7,8,18,19]$, and the next step is numerical calculation. However, the aims of the two steps are mixed together rather than clearly defined separately.

Many stem diameter retrieval numerical methods have been introduced and applied with different tree species and environmental factors during the past two decades. In summary, these methods can be roughly divided into two types. (1) The first type is classic regular approaches. The stem is simplified into a regular geometry, in which the stem cross-section is assumed to be a circular geometry. Therefore, the stem diameter is retrieved using CYF [9], CF [3], Hough transform [22] and variant methods [13,23]. These methods are easy to apply and can also output satisfactory results when the stem point density is insufficient, especially for single-scan data. However, these methods overlook the irregularity in the stem cross-section that can be derived from TLS data and may not always correspond to reality [7]. (2) The second type is simulated manual measurement approaches. The irregularity in the stem cross-section and the working scenario of the field work, i.e., the path of the diameter tape that is passed through the convex part of the stem cross-section, are considered in stem diameter retrieval. From this, the stem diameter is retrieved by simulating the path of the diameter tape using convex hull line fitting (CLF) [21,24], closure B-spline curve fitting (SP) [8] and closure Bézier curve fitting with global convexity (SPC) [18]. These methods consider irregularity of the stems, however, they are time-consuming and require high point densities. However, in different studies, the above methods have yielded satisfactory results with the respective study materials. It is difficult to directly compare the performances of these methods between different studies as the inputs are different $[7,12]$. This raises the question of how to choose a stem diameter retrieval numerical method, or how to retrieve stem diameter with high accuracy for a given stem point cloud.

In addition to the stem diameter tape, the caliper is a common measuring tool for stem diameter in the field work. The caliper is used to measure stem diameters in several directions, and the average of all the directional diameters is the measured stem diameter. Van Laar and Akca listed the detailed quality specifications for caliper operation [25]. The stem diameter measured with the diameter tape was slightly larger than that measured with the caliper in practice [26]. Tang theoretically proved that the average stem diameter measured in all directions with the caliper is equal to that measured using stem diameter 
tape whether the shape of the stem cross-section is convex or concave [27]. At present, TLS can capture the geometrical characteristics of the stem cross-section; thus, it is possible to evaluate the equivalency between the stem diameter tape and the caliper using TLS data by simulation measurements.

In this study, we consider the similarities and differences between several stem diameter numerical calculation methods without regard to the stem point preprocessing step. The specific objectives are (1) to practically and theoretically evaluate the performances of different stem diameter retrieval numerical methods, (2) to evaluate the equivalency between the diameter tape and the caliper, and (3) to discuss potential ways to improve the accuracy of stem parameter retrieval.

\section{Materials and Methods}

\subsection{Study Site and Field Data Collection}

The scanning sample and field-measured stem diameters were obtained in July 2018 in Dagujia Forest Farm (N 42 $21^{\prime}$, E 124 $52^{\prime}$ ) in Qingyuan County, Liaoning Province, China. The study materials were sourced from three Larix kaempferi plantation plots. The area of each plot was $30 \mathrm{~m} \times 30 \mathrm{~m}$, and the ages of the plots were 25, 36 and 36 years in 2018 . In the field work, the DBH was manually measured using stainless steel diameter tape according to a measuring position, which was labelled using white paint after peeling the bark in 2017 (Figure 1). The maximum, minimum and average values of the field-measured stem diameters were $34.8 \mathrm{~cm}, 11.7 \mathrm{~cm}$ and $19.2 \mathrm{~cm}$, respectively.

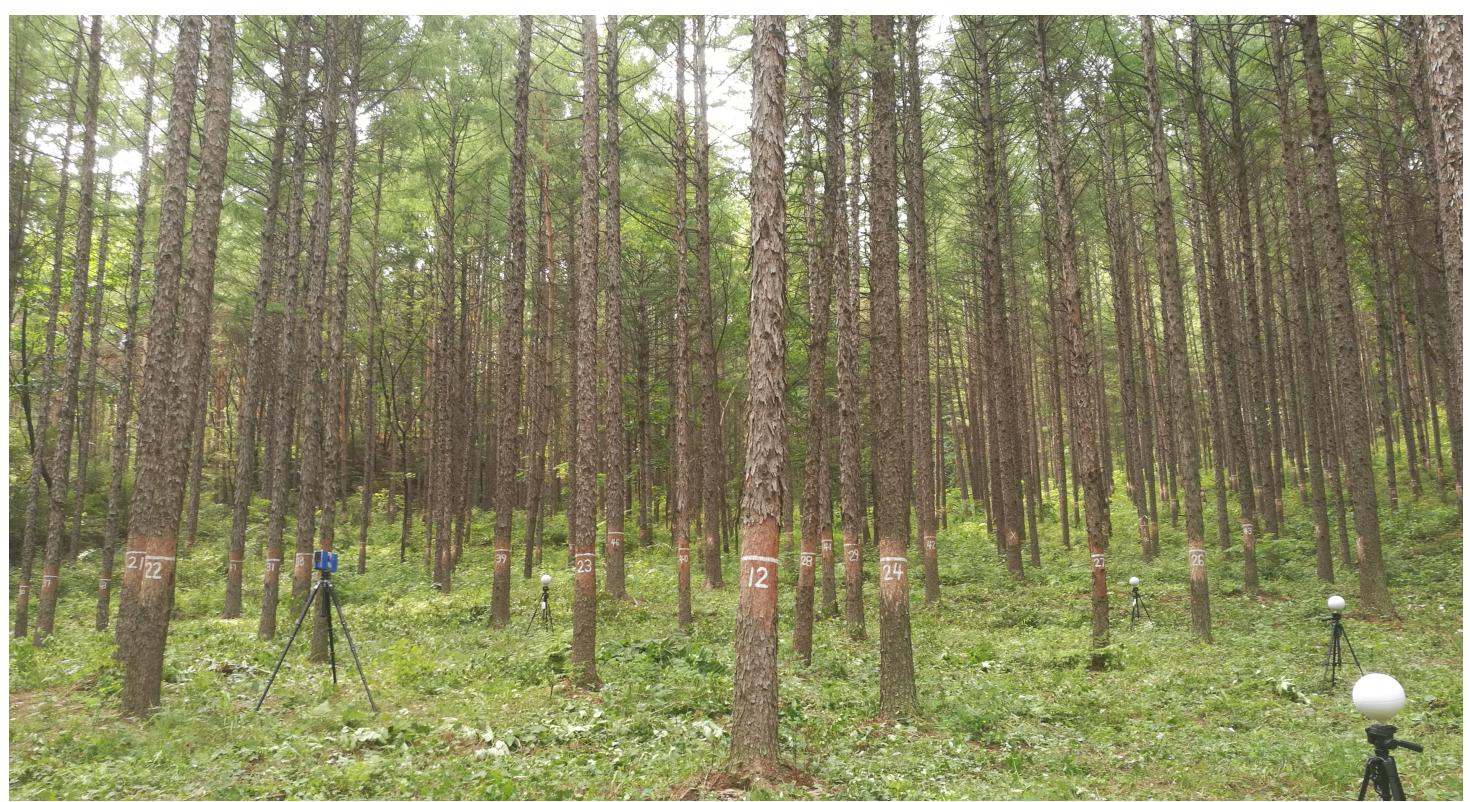

Figure 1. An image of the field work.

Trees were scanned from nine TLS stations (the center position of the stand, and the four corners and center positions of each border). The scanner device was a FARO Focus ${ }^{3 D}$ $X 330$ [28]. The scan quality was $4 \times$ (a scanning parameter of the FARO TLS scanner), the scan speed was $122 \mathrm{kpts} / \mathrm{s}$, the point spacing was $6.136 \mathrm{~mm}$ at $10 \mathrm{~m}$, and reference spheres were used for registration. Point cloud registration was performed using the FARO Scene 5.0 software, and the average of mean tension of each registration (the lower the tension value, the better the registration result) for three plots were $0.00261,0.01036$ and 0.00574 , respectively. After that, the single tree points were extracted according to the continuity of the stem in the vertical direction [29]. There were 65, 50 and 50 single trees extracted from the three plots. 


\subsection{TLS Data Preprocessing}

A set of data processing procedures was used to obtain the experimental data. For a tree point cloud, the outliers, for which the number of neighbor points in a radius of $1 \mathrm{~cm}$ was less than 2, were removed. After that, the 3D stem axis curve [18] represented by a cubic B-spline curve at a height range of $20 \mathrm{~cm}$ to $300 \mathrm{~cm}$ was constructed. Then, the stem growth direction, i.e., the normal vector of the stem cross-sectional plane, and the stem cross-sectional plane within the height range along the stem could be calculated by the constructed stem axis curve. In other words, for a given stem height with in the height range, the stem cross-sectional plane at the given height can be calculated. Therefore, the stem cross-sectional plane, which was located at the height at breast labeled by white paint (Figure 2), was calculated by the stem axis curve by searching for a suitable value for the height variable. After that, a stem slice was formed by two parallel stem cross-sections called the lower stem cross-section and the upper stem cross-section. As the impact of the accuracy of DBH retrieval was not significant when the thickness of the stem cross-section was 1-10 cm [19], the lower (upper) section was parallel to and below (above) the stem cross-sectional plane at breast height with a distance of $2.5 \mathrm{~cm}$ in this study. Thus, a stem slice with a length of $5 \mathrm{~cm}$, i.e., stem points between the two constructed stem cross-sections, for a single tree was extracted. Then, for a stem slice, a 3D stem slice point cloud was obtained through geometric transformation by rotating the vector from the stem growth direction at breast height to the vector $(0,0,1)$.

(a)

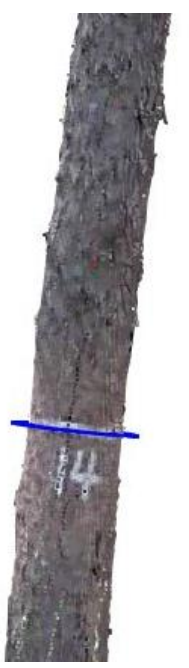

(b)

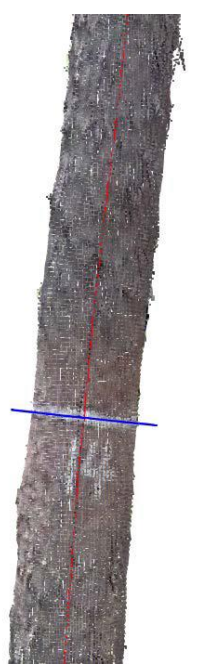

Figure 2. The calculated stem cross-sectional plane at breast height. (a) The diagram of a stem cross-sectional plane location by the stem axis curve, (b) the enlarge view of (a). The blue curve represents the stem cross-sectional plane, and the red curve in (b) is the constructed stem axis curve.

In this study, 165 3D stem slice point clouds were collected from 165 single trees. The stem diameter retrieval methods were separately applied to each 3D stem slice point cloud.

\subsection{Stem Diameter Retrieval Methods}

\subsubsection{Method Simulation for the Diameter Tape}

The initial inputs of the CYF, CF, CLF, CSM, SP and SPC methods were 3D stem slice point clouds, and different methods used different data derived from the 3D stem point clouds in the final calculation.

The final input of the CYF method was the 3D stem slice point cloud. As the stem growth direction of the 3D stem slice point cloud was rotated to the vector $(0,0,1)$ through geometric transformation, the stem growth direction, as well as the axis direction of the fitted cylinder, was equal to the vector $(0,0,1)$. Then, the stem diameter was retrieved by CYF using the least squares method [30], which uses the axis direction as an input parameter. 
The final input of the CF method was the two-dimensional(2D) stem slice point cloud, which was the projection point set of the 3D stem slice point cloud on the horizontal plane. Then, the stem diameter was retrieved by CF using the least squares method.

The final input of the CLF method was the convex hull point set, which is the convex hull of the 2D stem slice point cloud. Then, a convex hull polygon was obtained by connecting the two adjacent convex hull points. The stem diameter was equal to the perimeter of the convex hull polygon divided by the PI.

The final input of the SP method was the convex hull point set. A closed cubic B-spline curve was interpolated on the convex hull point set using global interpolation [31]. The stem diameter was equal to the length of the closure B-spline curve divided by the PI.

The final input of the SPC method was the convex hull point set. A closed convex cubic Bézier curve was obtained by constructing piecewise cubic Bézier curves with second-order geometric continuity and global convexity [32]. The stem diameter was equal to the length of the closure convex curve divided by the PI. Diagrams of the above methods with a stem slice point cloud are shown in Figure 3.
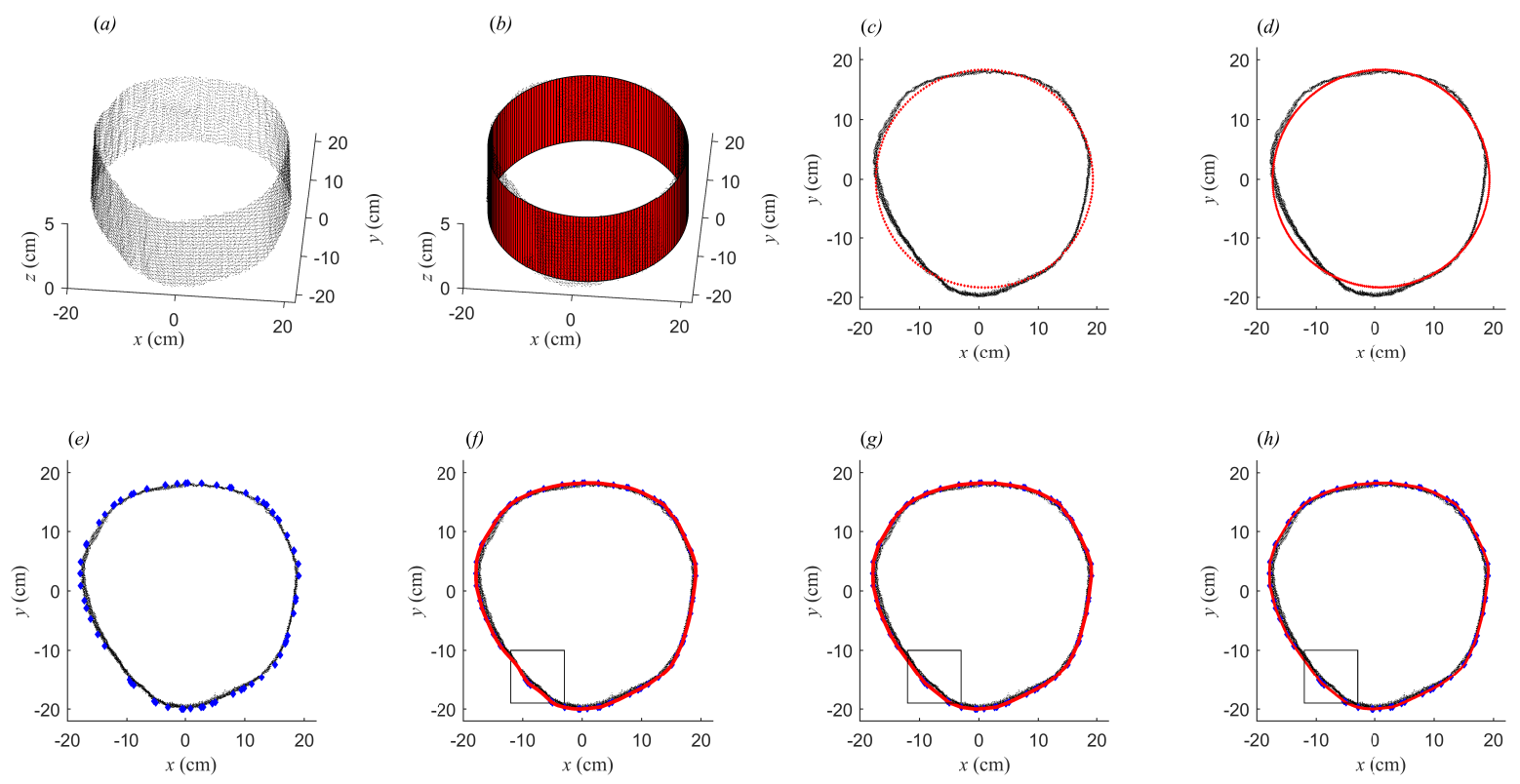

Figure 3. Diagrams of stem diameter retrieval methods using a stem slice point cloud with an ovality (Section 2.3.2) of $6.69 \%$. (a) A 3D stem slice point cloud; (b) CYF in 3D space; (c) the projection of the 3D stem slice point cloud and fitting points by CYF; (d) CF; (e) the projection of the 3D stem slice point cloud and its convex hull points; (f) SP; (g) CLF; (h) SPC. The rectangles in $(\mathbf{f}-\mathbf{h})$ represent the distinction part for the corresponding three methods. The black points represent stem points in $3 \mathrm{D}$ space(or their projection in 2D space), the blue points represent convex hull points, the red surface in (b) represents the surface of the fitted cylinder, and the red points (curve) represent the fitted points (curve).

\subsubsection{Method Simulation of the Caliper}

To the best of our knowledge, there have been no reported studies using caliper simulation to retrieve stem diameter using TLS data. In this study, we presented a caliper simulation method (CSM) to retrieve stem diameter using TLS data. The final input of the CSM method was the convex hull point set. In the field work, the two arms of the caliper were parallel and located on the convex part of the stem cross-section in each measurement direction. Thus, the two arms could be seen as two parallel lines that just passed through the convex part of the stem cross-section, while the entire region of the stem cross-section was located between the two lines. To ensure the accuracy of the CSM method, the convex hull point set was divided into $k$ (72 regions in this study) angular regions by the angle interval $\alpha$ (set to 5 in this study) degrees, and the angle value $\eta$ between convex hull points and the geometrical central point of the convex hull point set. Thus, convex hull points 
fell into different angle regions. The $i$-th region and $\left(i+\frac{360}{2 \alpha}\right)$-th region $\left(0 \leq i \leq \frac{360}{2 \alpha}-1\right)$ formed the $i$-th caliper measurement direction. Then, the caliper measurement could be simulated $\frac{360}{2 \alpha}$ times. For $i$-th direction, two lines were constructed to simulate the two arms of the caliper (Figure 4). The direction vector of the two lines was $\left(\sin \left(i \times \alpha+\frac{\alpha}{2}\right)\right.$, $\left.\cos \left(i \times \alpha+\frac{\alpha}{2}\right)\right)$.

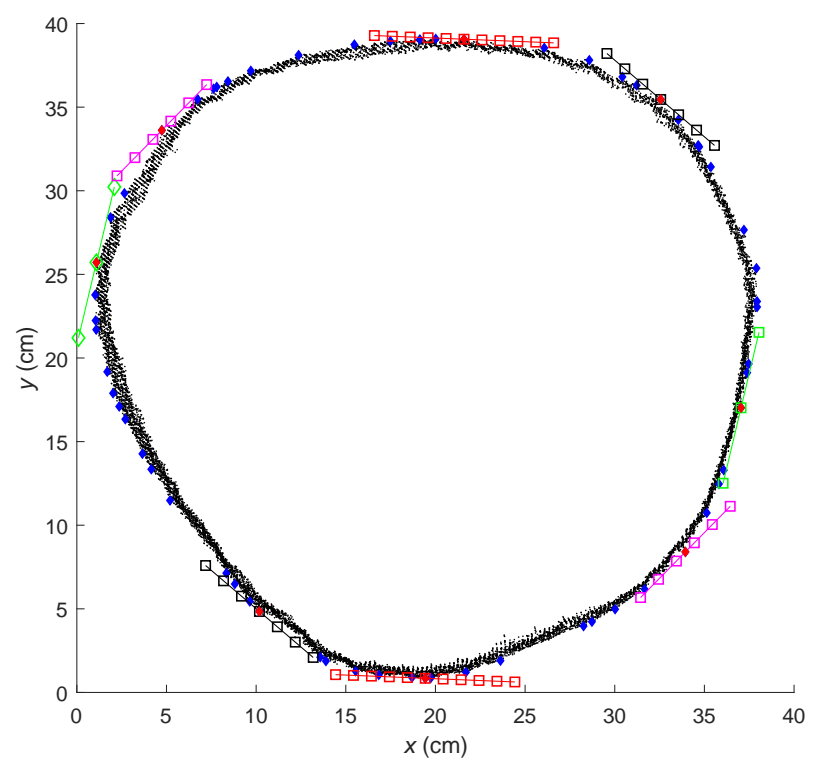

Figure 4. The diagram of the CSM. The black points represent the $2 \mathrm{D}$ stem slice points. The blue diamond points represent the convex hull points of the 2D stem slice point cloud. The colored lines with squares represent the simulated caliper arms, and the two lines in one direction are described in the same color. The red diamond points represent the two convex hull points that the two lines pass through.

After that, the convex hull point set was searched for the two convex hull points to ensure that the two lines passed through the two identified convex hull points and that the other convex hull points were located between the two lines. Then, the distance of the two lines was the stem diameter of the $i$-th region by caliper simulation. Finally, the final stem diameter, i.e., the average value of the stem diameters in $\frac{360}{2 \alpha}$ directions, was calculated after caliper simulation in each direction.

Based on the CSM method, the maximum and minimum stem diameters were retrieved from the $\frac{360}{2 \alpha}$ direction measurements. Thus, the ovality in percent [33] for a 3D stem slice point cloud was calculated according to the following formula.

$$
O=\left(1-\frac{D_{\text {Min }}}{D_{\text {Max }}}\right) \times 100
$$

where $D_{\mathrm{Min}}$ and $D_{\mathrm{Max}}$ represent the minimum and maximum stem diameters measured from the $\frac{360}{2 \alpha}$ directions.

\subsection{The Completeness of a Stem Slice Point Cloud}

The more complete a stem slice point cloud is, the clearer the geometry information of the real stem slice and the more the persuasive the retrieved stem parameters. It is hoped that the stem points to be evenly distributed around the center of the stem slice point cloud. However, due to various fielding work factors, not all the point clouds scanned by TLS can meet this demand. The completeness $C$ of a stem slice point cloud according to the angular regions of convex hull point set in Section 2.3.2, is presented in this study. The 2D stem slice point cloud was divided into $k$ angular regions with an angle interval $\alpha$ (set to 5 in this study), and meeting $k=\frac{360}{\alpha}$. The number of nonempty angular regions $k_{\mathrm{n}}$ was 
initialized to zero; if the $i$-th $(0 \leq i<k)$ angular region was is a nonempty angular region, i.e., at least one point was in the current angular region, the number of nonempty angular regions $k_{\mathrm{n}}$ increased by one. Then, the completeness $C$ of a stem slice point cloud could be calculated as follows.

$$
C=\frac{k_{\mathrm{n}}}{k} \times 100
$$

\subsection{The Roughness of a Stem Slice Point Cloud}

The internal points of a stem slice point cloud were used in the CYF and CF methods; however, these points were not used in the CLF, CSM, SP and SPC methods. It is necessary to know the distance information between the innermost point and the outermost point of a stem slice point cloud. In this study, the roughness of a stem slice point cloud is presented to represent the distance information. Based on the angular regions from Section 2.3.2, for the $i$-th nonempty angular region $t_{i}(0 \leq i<k)$ in a stem slice point cloud, the maximum and minimum distances between the geometrical central point and $t_{i}$ angular region points were first calculated, and then the roughness $r_{i}$ of $t_{i}$ was equal to the difference between the maximum distance and minimum distance. The roughness $R$ of a stem slice point cloud was the average of $r_{i}$ from nonempty angular regions.

$$
R=\frac{\sum\left(r_{i}\right)}{k_{\mathrm{n}}}
$$

Notably, a stem slice point cloud cannot fully reflect the real case of a stem slice due to the influence of occlusion and other scanning factors. Hence, the ovality, completeness and roughness here belonged to a stem slice point cloud rather than a real stem slice. Obviously, there was a gap between the ovality of the stem slice point cloud and that of the real stem slice when there were missed stem points in the stem slice point cloud. For example, a stem slice point cloud was sourced only from TLS single-scan data; in this case, the completeness of the stem slice point cloud was not more than 50\%. However, when the completeness of the stem slice point cloud was $100 \%$, the ovality of the stem slice point cloud could be seen as the ovality of the real stem slice.

\subsection{Assessment Indices}

The accuracy of stem diameter retrieval was evaluated using the mean error (ME), mean absolute error (MAE), mean absolute percentage error (MAPE), root mean square error (RMSE), and relative root mean square error (RRMSE).

\section{Results}

\subsection{The Basic Information of 3D Stem Slice Point Clouds}

The maximum, minimum and average values of ovality, completeness and roughness of the studied stem slice point clouds are listed in Table 1, and the histograms of ovality, completeness and roughness are shown in Figure 5.

Table 1. The statistical information of the studied 3D stem slice point clouds.

\begin{tabular}{cccc}
\hline & Ovality (\%) & Completeness (\%) & Roughness (cm) \\
\hline Maximum & 49.30 & 100.00 & 0.92 \\
Minimum & 2.60 & 55.60 & 0.15 \\
Average & 8.80 & 94.00 & 0.37 \\
\hline
\end{tabular}

The ovality values of the stem slice point clouds were mainly distributed between $5 \%$ and $10 \%$, and a few values were scattered. This means that there were certain degrees of differences between the minimum stem diameter and the maximum stem diameter for most stem slice point clouds, but there were also large differences for a few stem slice point clouds. The completeness values of the stem slice point clouds were mainly 
distributed between $95 \%$ and $100 \%$, and there were a small number of values below $75 \%$. This means that there were points on each angular region for most stem slice point clouds. The roughness of the stem slice point clouds was mainly distributed between $0.2 \mathrm{~cm}$ and $0.5 \mathrm{~cm}$. This means that the convexity or concavity of the stem slice point clouds was not severe.

(a)

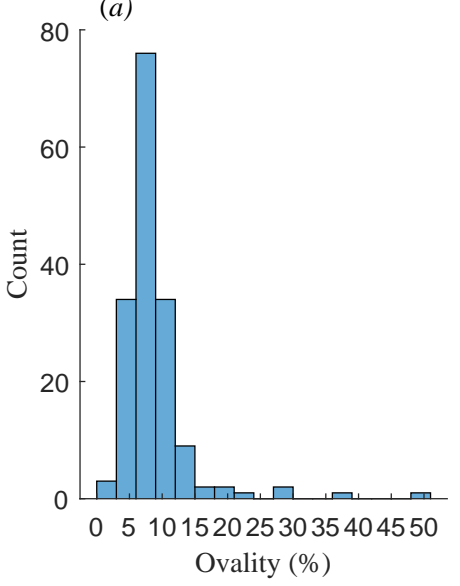

(b)

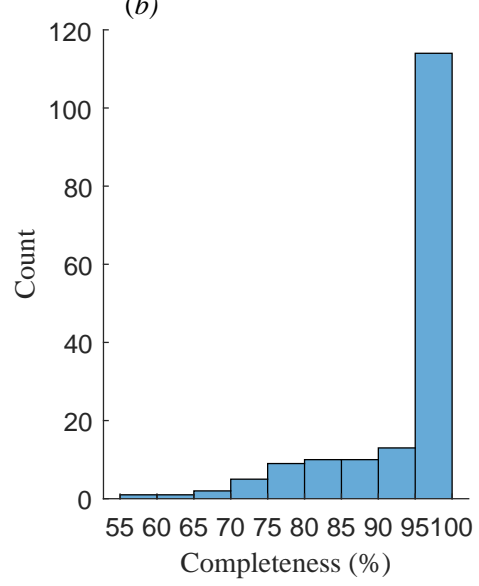

(c)

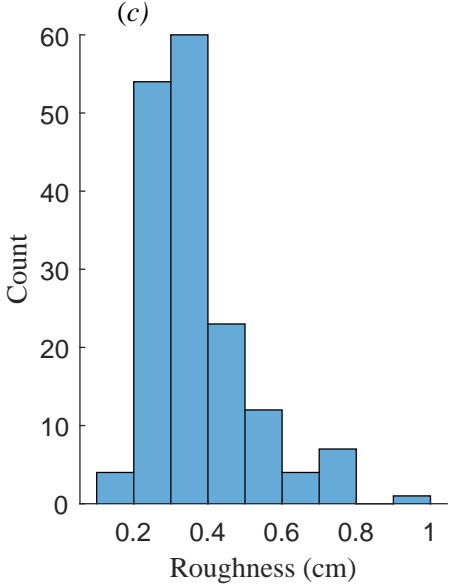

Figure 5. Histograms of the ovality (a), completeness (b), and roughness (c) of the stem slice point clouds.

According to Table 1 and Figure 5, the quality of the studied stem slice point clouds in this study can be described as follows. The average ovality was $8.80 \%$, demonstrating that most stem slice point clouds were relatively regular, i.e., that most of them were close to a circle; the average completeness was $94.00 \%$, demonstrating that most of the stem slice point clouds were relatively complete, and could effectively reflect the geometric structure of stem slices; the average roughness was $0.37 \mathrm{~cm}$, demonstrating that the local undulation degrees of most of the stem slice point clouds were small, i.e., that the stem surfaces of most of the stem slice point clouds were smooth. The data quality of the studied stem slice point clouds was good.

\subsection{Results of the Stem Diameter Retrieval Methods}

The differences between the field-measured DBH values and those of the stem diameter retrieval methods are shown in Figure 6, and the regression equations and the assessment indices are shown in Figure 7.

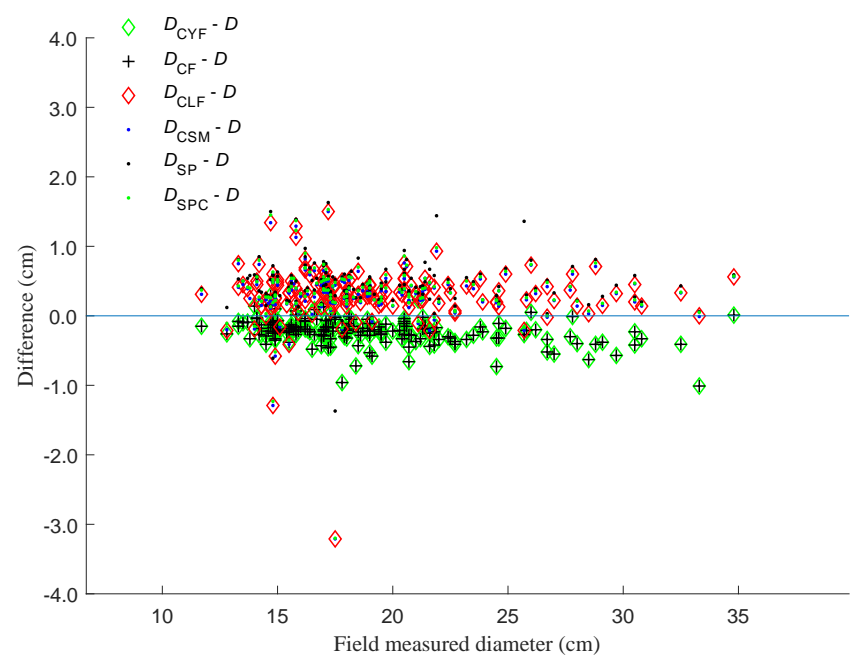

Figure 6. DBH differences between field-measured and stem diameter retrieval methods. $D_{\mathrm{CYF}}, D_{\mathrm{CF}}$, $D_{\mathrm{CLF}}, D_{\mathrm{CSM}}, D_{\mathrm{SP}}$, and $D_{\mathrm{SPC}}$ represent the $\mathrm{DBH}$ values retrieved by the CYF, CF, CLF, CSM, SP and SPC methods. $D$ represents the field-measured DBH. 
(a)

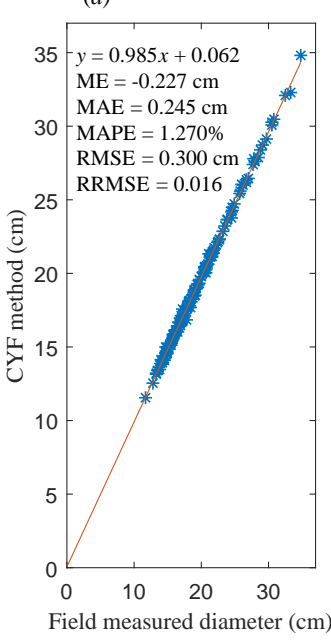

(d)

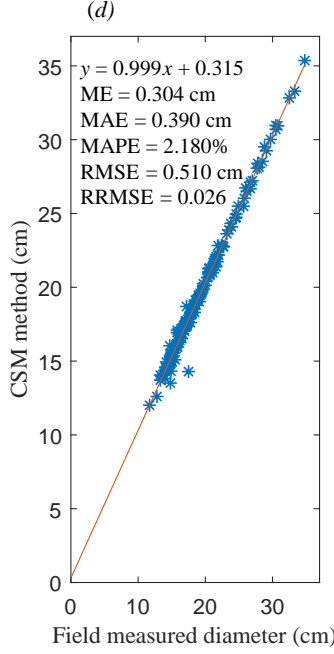

(b)

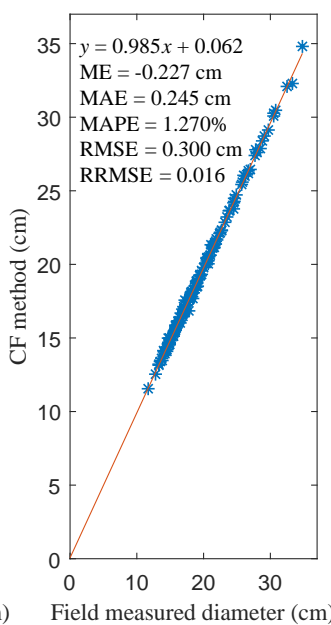

(e)

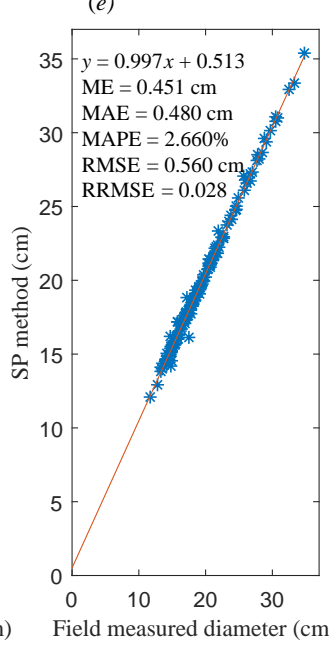

(c)

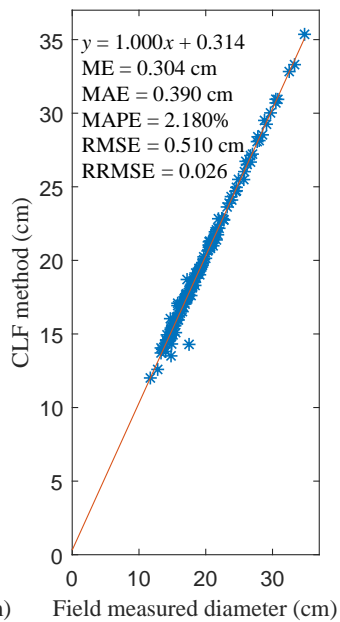

$(f)$

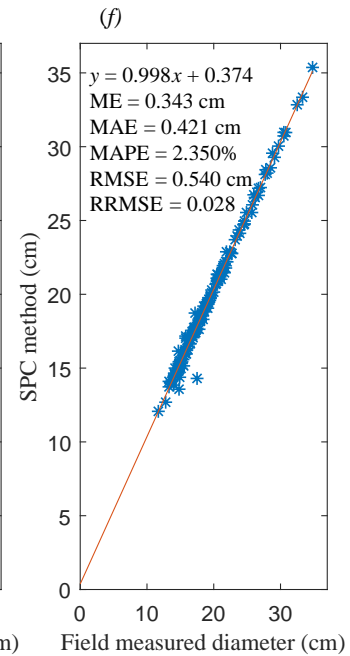

Figure 7. Regression equation and assessment index values between the field-measured DBH values and DBH values retrieved by the CYF method (a), CF method (b), CLF method (c), CSM method (d), SP method (e) and SPC method (f).

For the CYF and CF methods, most DBHs retrieved by the two methods were smaller than the field-measured DBHs, and the differences were mainly distributed between $-1 \mathrm{~cm}$ and $0 \mathrm{~cm}$ (Figure 6). Compared with the field-measured $\mathrm{DBH}$, the two methods have the same performance in terms of assessment indices (Figure 7). This means that the difference between the CYF method and field measurement was equal to that between the CF method and field measurement; i.e., the stem diameters retrieved by the CYF method and the CF method were identical in this study.

For the CLF, CSM, SP and SPC methods, most DBHs retrieved by the four methods were larger than the field-measured DBHs, and the differences were mainly distributed between $0 \mathrm{~cm}$ and $1 \mathrm{~cm}$ (Figure 6). Compared with the field-measured DBH, the CLF and CSM methods had very similar performances in terms of assessment indices(there was only a slight difference in the regression equations) (Figure 7). This means that the performances of the CLF and CSM methods were very similar in this study.

Parts of the assessment index values were list in Table 2 for ease of viewing. According to Figures 6 and 7 and Table 2, the performances of the CYF and CF were the best, with RMSE values of $0.30 \mathrm{~cm}$; the performances of the CLF and CSM were second, with RMSE values of $0.51 \mathrm{~cm}$; the performances of the SPC method was the third, with an RMSE value of $0.54 \mathrm{~cm}$; and the performance of the SP method was the worst, with an RMSE value of $0.56 \mathrm{~cm}$. In most cases, the DBHs retrieved by the CYF and CF methods were smaller 
than those retrieved by the other four methods; the DBHs retrieved by the CLF and CSM methods were smaller than those retrieved by the SP and SPC methods; and the DBHs retrieved by the SPC method were smaller than those retrieved by the SP method.

Table 2. Parts of the assessment index values of the six methods for stem diameter retrieval.

\begin{tabular}{cccc}
\hline Method & ME $(\mathbf{c m})$ & MAE $(\mathbf{c m})$ & RMSE $(\mathbf{c m})$ \\
\hline CYF & -0.227 & 0.245 & 0.300 \\
CF & -0.227 & 0.245 & 0.300 \\
CLF & 0.304 & 0.390 & 0.510 \\
CSM & 0.304 & 0.390 & 0.510 \\
SP & 0.451 & 0.480 & 0.560 \\
SPC & 0.343 & 0.421 & 0.540 \\
\hline
\end{tabular}

The relationship between the ovality, completeness, and roughness of stem slice point clouds and the retrieved DBH difference are shown in Figures 8-10.

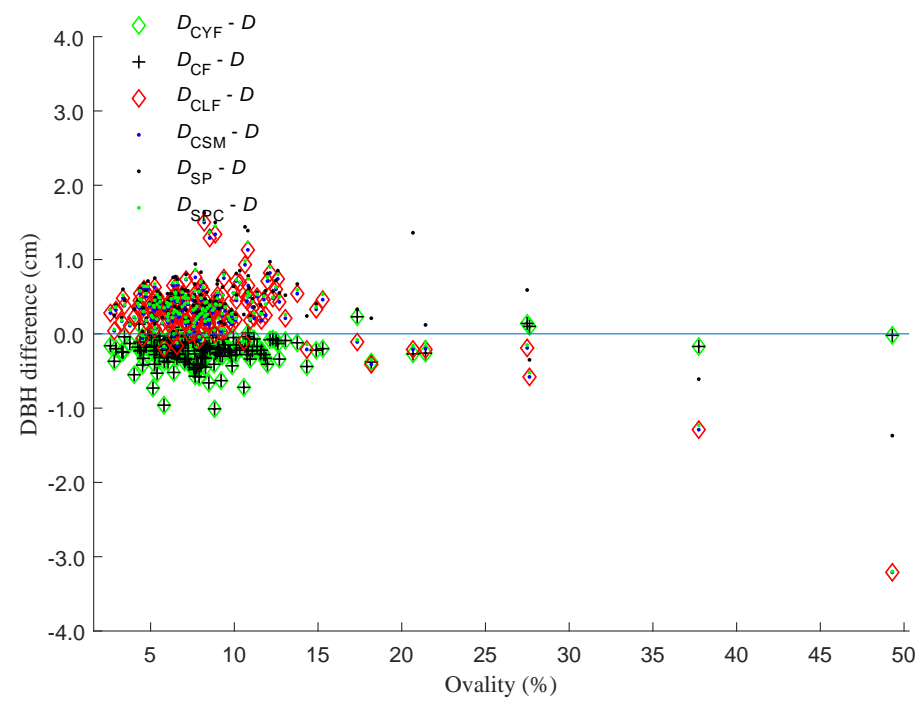

Figure 8. The relationship between the ovality of the stem slice point clouds and DBH differences.

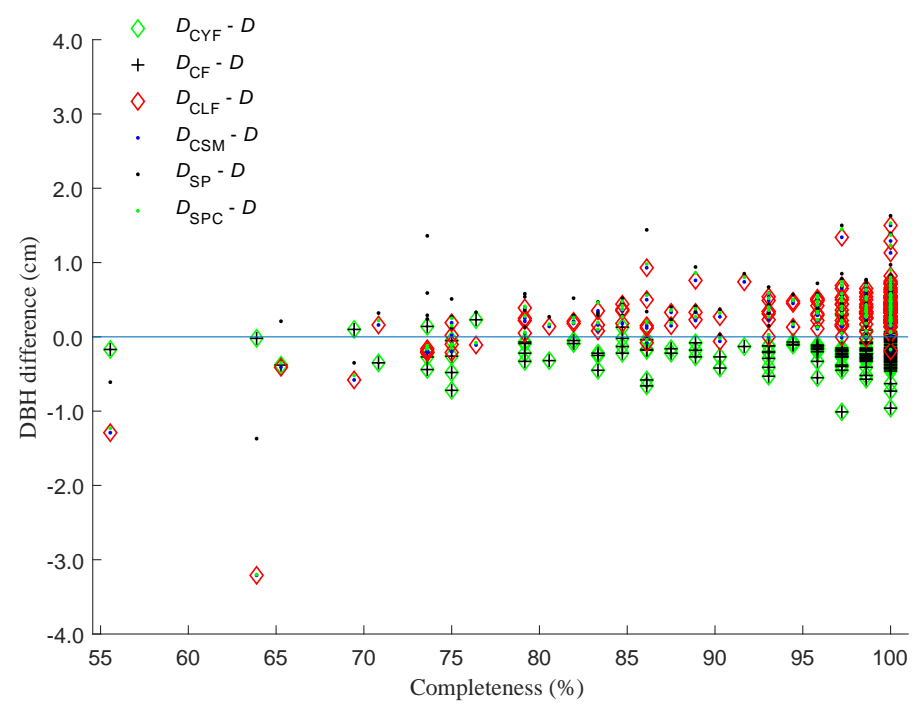

Figure 9. The relationship between the completeness of the stem slice point clouds and DBH differences. 


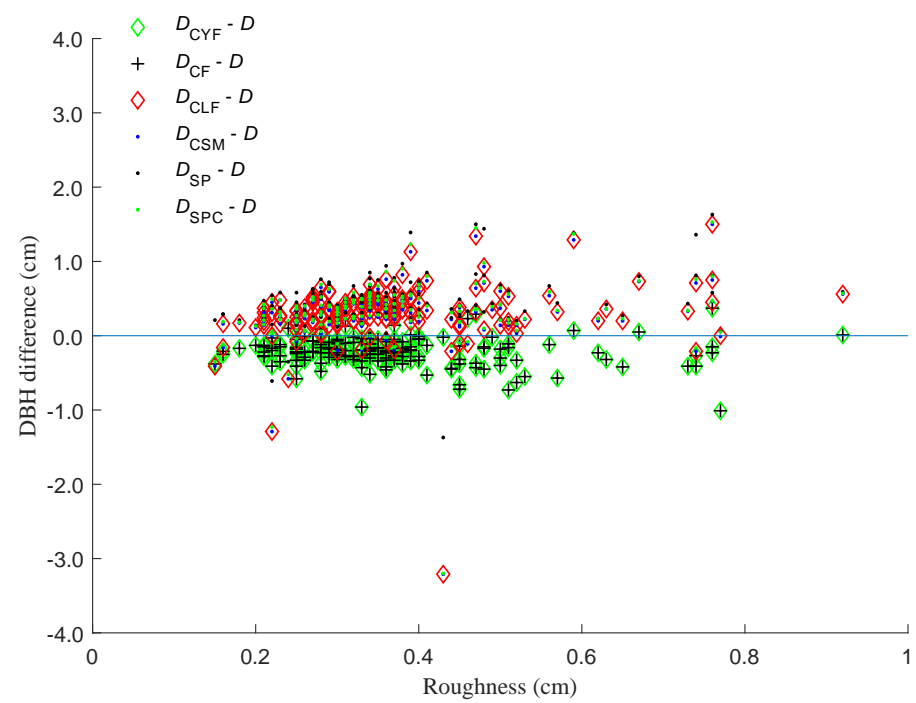

Figure 10. The relationship between the roughness of the stem slice point clouds and DBH differences.

From Figure 8, when the ovality of the stem slice point cloud is high, the DBHs retrieved by the CLF, CSM, SP and SPC methods were smaller than the field-measured DBHs, and the difference between the two DBHs tended to increase with ovality. The difference between DBH retrieved by the CYF and CF methods and the field-measured DBH did not increase with the ovality in this study.

From Figure 9, when the completeness of the stem slice point cloud was less than $70 \%$, the DBHs retrieved by the CLF, CSM, SP and SPC methods were smaller than the fieldmeasured DBHs. For the CYF and CF methods, the relationship between the completeness of the stem slice point cloud and DBH differences was not obvious in this study.

From Figure 10, the relationship between the roughness of the stem slice point cloud and $\mathrm{DBH}$ differences was not obvious.

According to the above, the CYF and CF methods were not sensitive to the stem slice point clouds and were superior to the other four methods. Additionally, the ovality and completeness of the stem slice point cloud affected the accuracy of the retrieved DBH. Considering that the ovality is affected by the completeness of the stem slice point cloud, what is the accuracy of the retrieved DBH when the completeness of the stem slice point clouds is $100 \%$ ? A total of 68 stem slice point clouds with a completeness of $100 \%$ were used to calculate the regression equations and the assessment indices (Figure 11).

According to Figures 7 and 11, the robustness of the CYF and CF methods was demonstrated, as these assessment index values changed very little in two real cases; although the changes were mainly reflected in the MAPE and RRMSE, the accuracies of the CYF and CF methods were slightly improved; the ME and MAE values of the CLF, CSM, SP and SPC methods were increased, the RMSE and RRMSE values of the CLF, CSM and SPC methods were decreased, and the RMSE and RRMSE values of the SP method were increased. For the CLF, CSM, SP and SPC methods, the retrieved DBH values that obviously deviated from the regression line in Figure 7, i.e., the data that were more than $1 \mathrm{~cm}$ smaller than the field-measured DBH values in Figure 9, were eliminated. From this point of view, the accuracies of the CLF, CSM, SP and SPC methods were improved. The performances of these six methods in these two cases also demonstrated that the quality of the stem slice point cloud affected the accuracy of stem diameter retrieval. 
(a)

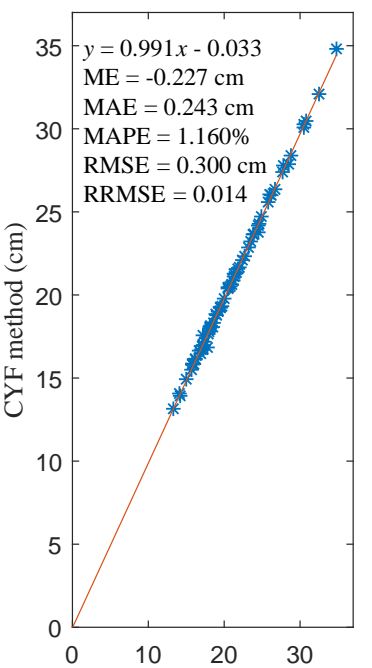

Field measured diameter $(\mathrm{cm})$ (b)

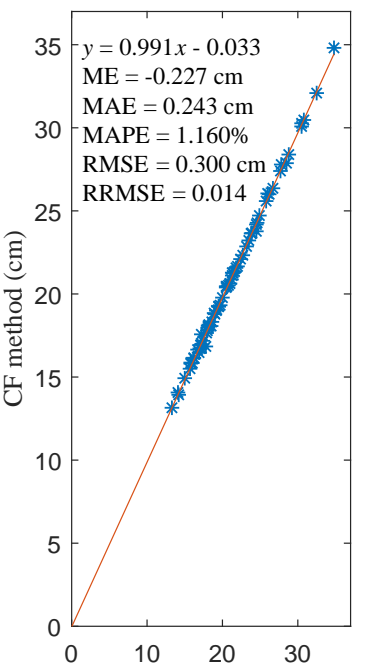

Field measured diameter $(\mathrm{cm})$ (c)

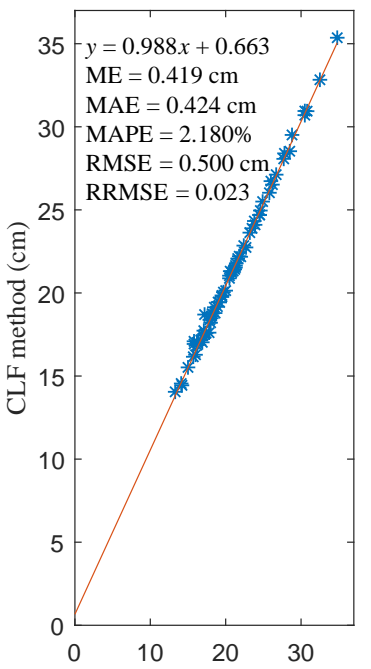

Field measured diameter $(\mathrm{cm})$
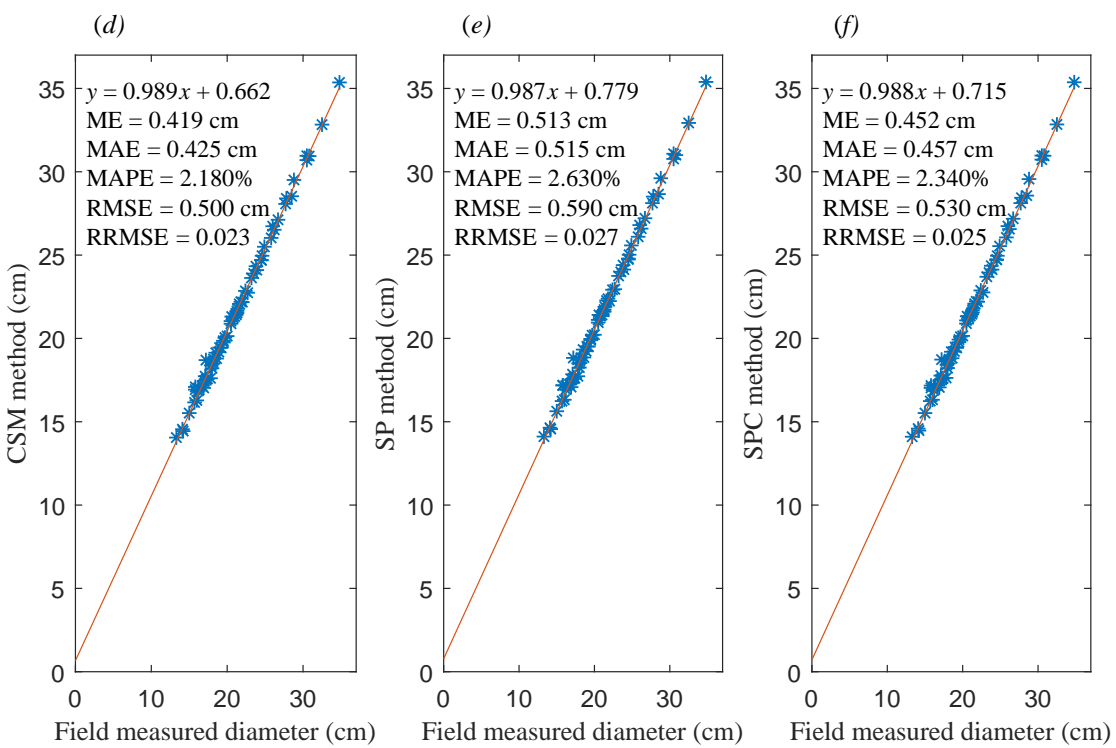

Figure 11. Regression equation and assessment index values between the field-measured DBH values and those of the CYF method (a), the CF method (b), the CLF method (c), the CSM method (d), the SP method (e), the SPC method (f) for stem slice point clouds with a completeness of $100 \%$.

\section{Discussion}

\subsection{Performance Analysis of Stem Diameter Retrieval Methods}

In this study, the original input of these methods was 3D stem slice point clouds collected from the stem cross-sections with the thickness of $5 \mathrm{~cm}$ by the 3D stem axis curve. Although the final input of these methods varied, the influencing factors from the use of different methods with different inputs were eliminated.

The final inputs of the CYF and CF methods were the 3D stem slice point cloud and the 2D stem slice point cloud, respectively. The 2D stem slice point cloud was the projection point set of the 3D stem slice point cloud. The normal vector of the projection plane was equal to the rotated stem growth of the 3D stem slice, as well as the axis direction of the fitted cylinder. The least squares method was used as the fitting method for the two methods, which was why the CYF and CF methods behaved the same way (Figure 3c,d) and yielded the same performance for stem diameter retrieval (Figure 7a,b). 
The convex hull point set in 2D space derived from the 2D stem slice point cloud were used as input for the CLF, CSM, SP and SPC methods in this study. The constructed curve by the SPC method and the constructed lines by the CLF method were closed with global convexity. The two parallel lines constructed by the CSM method went around the 2D stem slice point cloud from different directions, and the two parallel lines of each direction formed a convex region. The measurements from all directions formed an integrated convex region. This means that the CLF, CSM and SPC methods had very similar measurement modes, constructing a compact convex region to surround the 2D stem slice. Additionally, the curve constructed by the SPC method had second-order geometric continuity, while the CLF and CSM methods did not. Hence, the performances of the CSM and CLF methods were the same, and very similar to the performance of SPC. (Figure 7c,d,f).

Although the SP method interpolated on the convex hull points, the constructed cubic B-spline curve was not a global convex curve [8] (as the rectangle shown in Figure 3f). This caused that the performance of the SP method is worse than those of the CLF, CSM and SPC methods.

Most stem diameters retrieved by the CYF and CF methods were smaller than fieldmeasured stem diameters. That can be explained by the following two aspects. One aspect is that the CYF (CF) uses a 3D (2D) stem slice point cloud, while the field-measured stem diameter was extracted from the convex part of the stem cross-section under manual tension using stem diameter tape. The stem bark at breast height was peeled, and most of the turnup bark was removed. Hence, most of the 3D (2D) stem slice point clouds were located in the convex part of the stem cross-section formed in the field work. The other aspect is that the most of the curve fitted by the least squares method should be located between the innermost point and the outermost point in theory. According to the definition of the roughness of a stem slice point cloud in this study, the greater the roughness, the more irregular the local part of the stem slice point cloud and the larger the distance between the fitted curve and the innermost point and the outermost point (Figure 12).

Most stem diameters retrieved by the CLF, CSM, SP and SPC methods were larger than the field-measured stem diameters because manual tension eliminates the influence of turnup bark in field measurements. However, although peeling was applied in the field work and outliers were removed in preprocessing, the influence of a small turnup bark was not eliminated when the CLF, CSM, SP and SPC methods were used. Some sporadic convex points (the convex hull points in rectangles in Figure 12b) are very similar to turnup bark points, as they are not gradually protruding parts and there are inner points along the direction toward the center point. These points may have no effect in field measurements because they are compressed. However, these points are convex hull points in the CLF, CSM, SP and SPC methods.
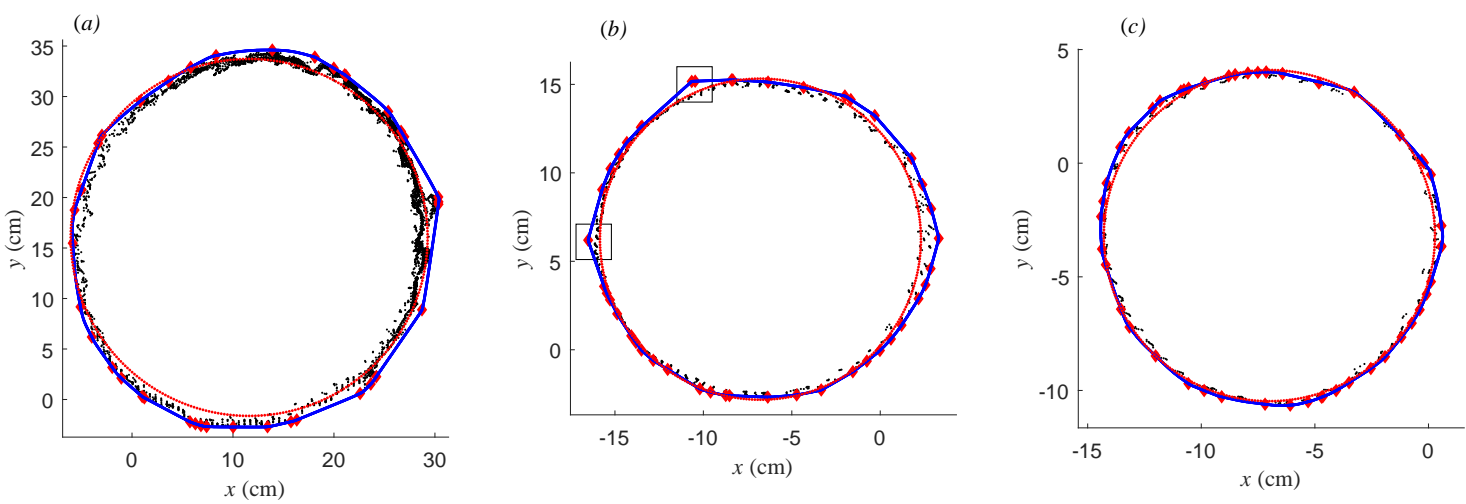

Figure 12. The results of the CF and SPC methods using stem slices with different roughness. The roughness values are (a) $1.23 \mathrm{~cm},(\mathbf{b}) 0.37 \mathrm{~cm}$ and (c) $0.20 \mathrm{~cm}$, respectively. The black points represent the 2D stem slice points, the red diamonds represent the convex hull points of the $2 \mathrm{D}$ stem slice point cloud, the red points represent the circle points fitted by the $\mathrm{CF}$ method and the blue curves represent the closure curve with global convexity by the SPC method. 


\subsection{The Equivalence of Stem Diameter Measurement between the Stem Diameter Tape and the Caliper}

The RMSE values between the field-measured DBH using the diameter tape and the CSM method simulating the caliper measurement in Figures 7 and 11 were $0.51 \mathrm{~cm}$ and $0.50 \mathrm{~cm}$, respectively. This demonstrates the approaching degree between the diameter tape and the caliper simulation.

Manual measurement with a caliper or diameter tape may lead to potential errors due to the operator's visual estimation [6]. Therefore, the DBH retrieved by simulation methods was used to evaluate the equivalence. Note that the elasticity between stainless steel stem diameter tape and fabric (plastic) stem diameter tape is different. The convex and smooth properties of the steel need to be ensured when using stainless steel stem diameter tape, which can be simulated by the SPC method. However, only the convex property needs to be ensured when using fabric (plastic) stem diameter tape, which can be simulated by the CLF method. Therefore, the SPC and CLF methods were used to represent the measurement using these two types stem diameter tape. Figure 7 shows that the CLF and CSM methods yield the same performance, and the regression equation and assessment indices between the SPC and CSM methods are shown in Figure 13.

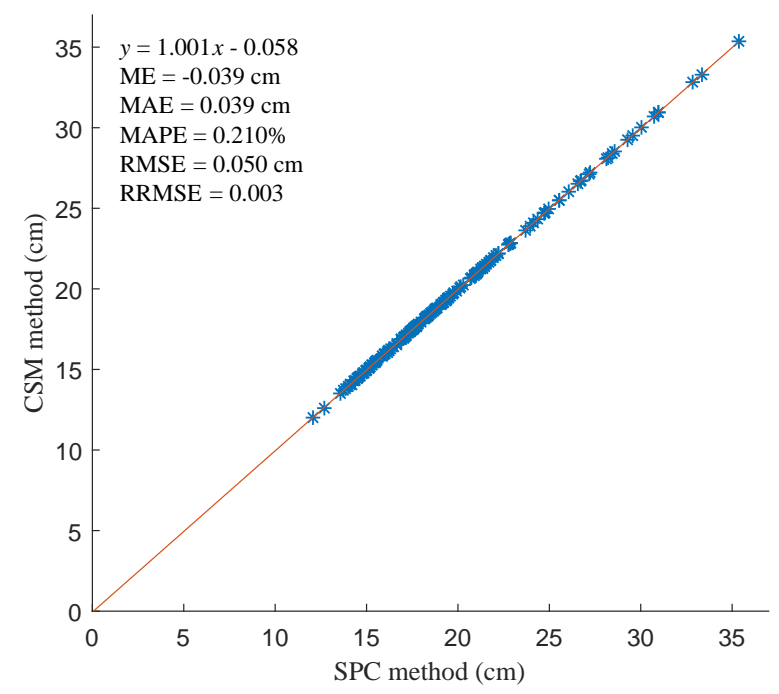

Figure 13. Regression equation and assessment index values between the SPC and CSM methods.

Compared with the SPC method, the ME of the CSM method was $-0.039 \mathrm{~cm}$, demonstrating that the DBH retrieved by the SPC method was slightly larger than that retrieved by the CSM method in this study. This result is in accordance with the conclusion in [26]. The RMSE of the CSM method was $0.05 \mathrm{~cm}$, which shows the high similarity between the two simulation measurement methods. Additionally, that the CLF and CSM methods performed the same is in accordance with the conclusion in [27]. Regardless of the kind of material of the stem diameter tape, the comparison between the SPC and CSM methods and the CLF and CSM methods demonstrates that the equivalent between stem diameter tape and caliper are acceptable for forestry measurements.

\subsection{The Usage of Ovality, Completeness and Roughness}

According to the definitions of ovality, completeness and roughness, the higher the completeness is, the lower the ovality and the lower the roughness. Meanwhile, the more complete the point cloud is, the more regular and the more smooth the stem slice point cloud. Therefore, the ovality, completeness and roughness of a stem slice point cloud can be used to evaluate the validity of the stem slice point cloud and the regularity of the stem slice (stem cross-section) using TLS data. In Figure 14a-c were sourced from the breast height, such as the DBH retrieval in this study, and others were sourced from other heights. 
It is easy to distinguish which stem slice point clouds can be directly used for parameter extraction, and which need further processing for parameter extraction. It is obvious that the stem slice point cloud in Figure 14c is suited for the CYF and CF methods rather than the CLF, CSM, SP and SPC methods.

(a)
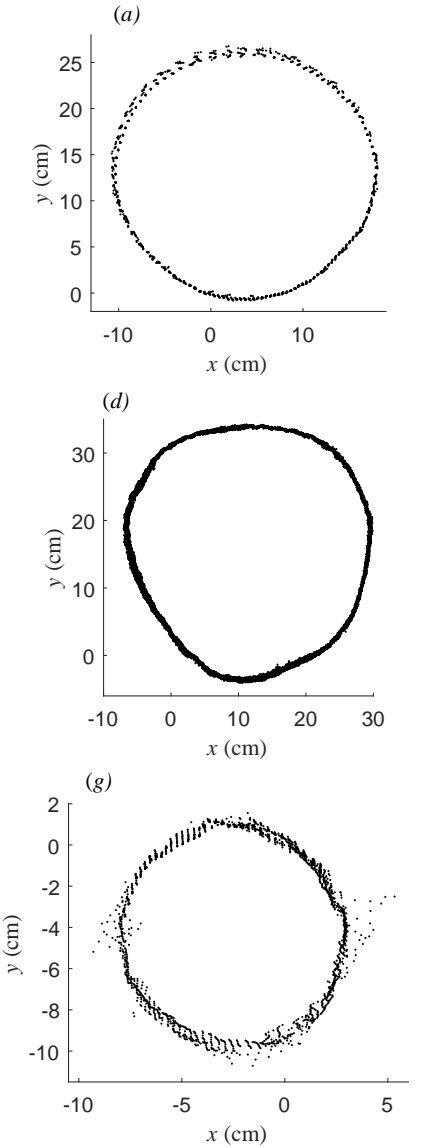

(b)

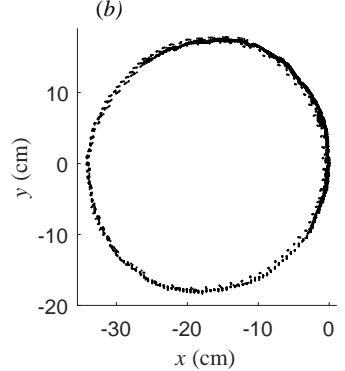

(e)

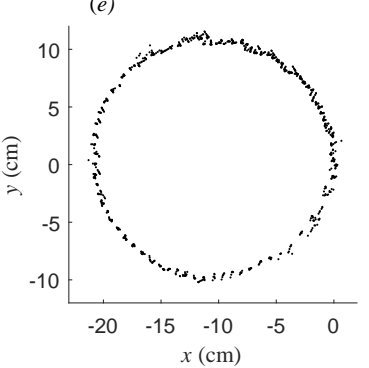

(h)

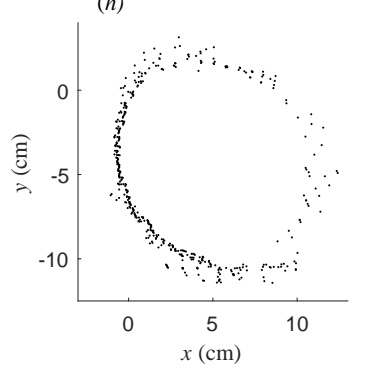

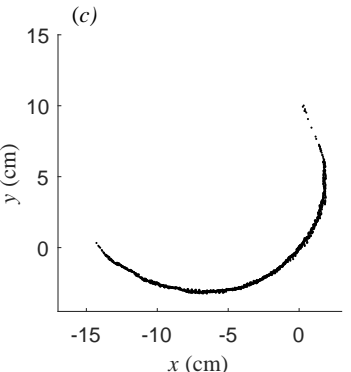

(f)

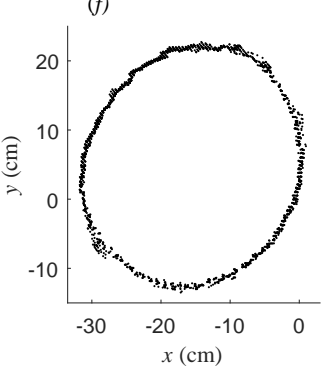

(i)

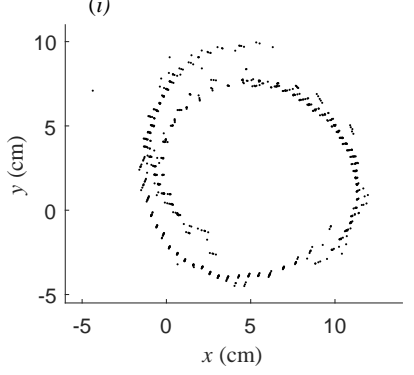

Figure 14. Nine stem slice point clouds with different values of ovality, completeness and roughness, where the values are (a) $5.96 \%, 100.00 \%$ and $0.50 \mathrm{~cm},($ b) $7.88 \%, 100.00 \%$, and $0.92 \mathrm{~cm},($ c) $49.30 \%, 63.00 \%$ and $0.43 \mathrm{~cm},(\mathrm{~d}) 6.69 \%, 100.00 \%$ and $0.86 \mathrm{~cm}$, (e) $5.07 \%, 100.00 \%$ and $0.41 \mathrm{~cm}$, (f) $15.01 \%, 100.00 \%$ and $0.88 \mathrm{~cm},(\mathrm{~g}) 14.85 \%, 100.00 \%$ and $0.75 \mathrm{~cm}$. (h) $20.94 \%$, $93.00 \%$ and $0.61 \mathrm{~cm}$ and (i) $26.29 \%, 100.00 \%$ and $1.10 \mathrm{~cm}$.

The ovality of a stem slice point cloud depends on not only the completeness of the stem slice point cloud but also the ovality of the real stem slice. When the completeness of the stem slice point cloud is $100 \%$ (or larger than $90 \%$ ), the ovality changes with the regularity of the real stem slice. The higher the ovality is, the higher the degree of irregularity of the stem slice (Figure 14a,b,e,f). When the ovality is greater than a given threshold value (such as $20 \%$ ), the validity of the stem slice point cloud starts to decrease (Figure 14h,i). It is easy to find that the two stem slice point clouds were registered incorrectly; in this case, a large error is unavoidable for all the above six stem diameter retrieval methods.

The influence of roughness was weakened because the stem bark around breast height was peeled in this study. The stem slice point cloud with the maximum roughness in this study is shown in Figure 14b. In fact, the maximum, minimum and average roughnesses at a height of $90 \mathrm{~cm}(150 \mathrm{~cm})$ of the studied stem were $1.13 \mathrm{~cm}, 0.20 \mathrm{~cm}$ and $0.50 \mathrm{~cm}$ $(1.51 \mathrm{~cm}, 0.21 \mathrm{~cm}$ and $0.67 \mathrm{~cm})$, respectively. The roughness differences between peeled and unpeeled stem parts can be observed in Figure 1. According to Section 4.1, the higher the roughness is, the larger the error between the retrieved DBH and field-measured DBH. High roughness in an angular region means that there are abnormal points in this region. Although the roughness of the stem slice point cloud in Figure 14g is not too high, 
there are high roughness in some angular regions, as there are two obvious outlier zones in Figure 14g.

The quality of a stem slice point cloud depends on a number of factors, such as the topographic conditions, tree density, ground vegetation, scanning number and scanning position. This also means that the trees in the same plot may obtain stem slice point clouds with different qualities. Hence, it is difficult to use the condition of the sample plot to describe the quality of each stem slice point cloud, although they are related. The above three variables provide quantitative methods for evaluating the quality of each stem slice point cloud.

\subsection{The Necessity and Importance of Stem Point Preprocessing}

The maximum and minimum RMSEs of the six stem diameter retrieval methods were $0.56 \mathrm{~cm}$ and $0.30 \mathrm{~cm}$ in this study. These values are smaller than those in most of the corresponding contemporary studies, such as the RMSE of $1.14 \mathrm{~cm}$ [34], RMSE values of $0.73 \mathrm{~cm}$ at $1.3 \mathrm{~m}$ and $0.84 \mathrm{~cm}$ at $6 \mathrm{~m}$ [7], RMSE of $1.17 \mathrm{~cm}$ [22], and RMSE ranging from 0.8 to $1.3 \mathrm{~cm}$ [3]. Additionally, the RMSE values of the six stem diameter retrieval methods were similar in this study. Although direct comparison of the accuracies from different studies is difficult because the input data vary $[7,12]$, the high and similar performance of the six stem diameter retrieval methods demonstrated that the differences between different stem diameter retrieval methods are not obvious when the same 3D stem slice point clouds are used. This means that the good quality of the studied 3D stem slice point clouds is a factor in the high accuracies of these stem diameter retrieval methods. Considering that stem point preprocessing will help to improve the quality of the stem point cloud, it is necessary to preprocess the stem slice point cloud before stem parameter retrieval.

The axis direction of the cylinder was an input parameter in the CYF method in this study. This is different from some exist CYF methods; for example, the axis direction is an output variable and is equal to the eigenvector corresponding to the maximum eigenvalue by principal component analysis (PCA) [35], which is suitable when the thickness of the stem slice point cloud is larger than the diameter. As the diameter of the fitted cylinder is closely related to the axis direction, the accuracy of the stem diameter can be ensured when an accurate axis direction is provided. For the CYF method in this study, the input 3D stem point cloud was geometrically transformed by rotating the stem growth direction to the vector $(0,0,1)$. Then, the vector $(0,0,1)$ seemed to be the new axis direction and an input parameter for CYF. For the CF and other methods in 2D space, the input was the projection of the transformed 3D stem point cloud. It is obvious that the stem growth direction is not only a parameter in data preprocessing in this study but also an important implicit parameter for stem diameter retrieval, which should be considered.

Fourteen DBH retrieval algorithms (seven algorithms based on the CF method, five algorithms based on the CYF method, one algorithm based on maximum distance, and another algorithm for which the details have not been published) were compared using identical TLS datasets, and the results were evaluated through a common procedure from reliable references [2]. These algorithms should yield similar performance, as the input data are the same and the numerical computation methods are very similar (most are based on CF and CYF methods). The study reported that the performances of these algorithms were different from each other, parts of the algorithms underestimated the $\mathrm{DBH}$, while the others overestimated the $\mathrm{DBH}$, and the differences between different algorithms were quite obvious. According to the performance of CF and CYF methods in this study, although the numerical computation methods of these fourteen algorithms have some subtle differences, the main difference between these fourteen algorithms should be the stem point preprocessing. Thus, the necessity and importance of stem point preprocessing is highlighted.

Although the CYF and CF methods are not sensitive to outliers, this does not mean that the influence of outliers can be overlooked in the CYF and CF methods. Outliers cause significant errors in the CYF [35] and CF [2] methods. The CLF, CSM, SP, and SPC methods are 
constructed based on convex hull points; these methods are more sensitive to outliers than the CYF and CF methods. The outliers in the stem slice point cloud in Figure 14g inevitably affects the accuracy of stem parameter retrieval. Therefore, regardless of the method used, removing outliers is a necessary condition for accurately retrieving stem parameters.

Obviously, the higher the quality of the stem slice point cloud is, the more accurate the retrieved stem parameters. The perfect stem slice point cloud should be able to effectively reflect the profile characteristics of the stem cross-section, and there should be no (or few) noise points. However, it is difficult to obtain such an ideal stem slice point cloud in sample plot scanning. Therefore, according to the ovality, completeness and roughness, evaluating and repairing the quality of the stem slice point cloud is a potential step for accurate stem parameter retrieval.

Most stem diameter retrieval algorithms include a stem point processing part, either indirectly or directly. For example, Wang et al. used a Fourier series curve to eliminate the outliers and branch points, and to approximate the stem cross-section shapes [20]. Stovall et al. used an outer hull model method to iteratively fit convex hulls and to handle noisy data [21]. Pitkänen et al. evaluated the stem cross-section with multiple-scan data using CYF and if it was found to be deficient, the diameter was detected using the single-scan data [7]. These above data processing parts are either mixed with or cooperate with the numerical calculation method. Separately obtaining the influence of subsequent calculation methods and accurately defining the aim of stem point processing helps to obtain stem slice point clouds with high quality, thereby improving the accuracy of stem parameter retrieval.

\subsection{A Potential Way to Improve the Accuracy of Stem Diameter Retrieval}

The stem growth direction and the thickness of the stem slice point cloud are important parameters that directly influence the accuracy of stem diameter retrieval. Therefore, the stem growth direction should be accurately calculated, and the thickness of the stem slice point cloud can be adjusted through stem slice point cloud quality assessment or experimental verification.

For a stem slice point cloud from a single scan or multiple scans, the quality can be first evaluated by the ovality and roughness. If the roughness or ovality exceeds its thresholds, the stem slice point cloud should be checked and processed to ensure that there are no noise points or that these points are correctly registered. Then, the stem diameter retrieval method can be chosen with the completeness. If the completeness is less than the threshold, the CYF or CF methods are recommended. When the completeness exceeds its threshold, considering that most stem diameters retrieved by the CYF or CF methods are smaller than the field-measured diameters and that most stem diameters retrieved by the CLF, CSM and SPC methods are larger than the field-measured diameters, the stem diameter can be represented by the average value (or linear combination ) of stem diameters retrieved from the CYF or CF method and the stem diameters retrieved from the CLF, CSM or SPC method. According to computational complexity, the order of selection priorities of the CYF and CF methods is the same, as the two methods are both implemented by the least square method; the order of selection priorities of the CLF, CSM and SPC methods is CLF, CSM and SPC. For the respective thresholds of ovality, roughness and completeness and processing methods for outlier removal and registration correction, further research is needed.

In addition to the above, some scanner errors need to be addressed to improve the accuracy of TLS scanning. For example, the laser point has a certain diameter, a laser beam may be fragmented, its reflection may be distorted, and the scanner will register two signals. The measurement result of TLS will different from the actual value, because noise or outliers will be generated during TLS working, and it is difficult to detect all the noises and outliers. 


\section{Conclusions}

We focused on the similarities and differences between stem diameter retrieval numerical methods using a set of 3D stem slice point clouds, that were extracted using a common preprocessing procedure to reduce the impact of data preprocessing. The CSM was presented to verify the equivalency between stem diameter tape and calipers for diameter measurement, and the ovality, completeness and roughness were presented to evaluate the quality of the stem slice point clouds. The results showed that the average ovality, completeness and roughness values of the studied stem slice point clouds were $8.80 \%, 94.00 \%$ and $0.37 \mathrm{~cm}$, respectively; the CYF and CF methods yielded the same performance, and the CLF and CSM methods yielded very close performance. Most stem diameters retrieved by the CYF and CF methods were smaller than field measurements, and most stem diameters retrieved by the CLF, CSM, SP and SPC were larger than the field measurements. Compared with the field-measured DBH, the RMSEs of the CYF, CF, CLF, CSM, SP and SPC methods were $0.30 \mathrm{~cm}, 0.30 \mathrm{~cm}, 0.51 \mathrm{~cm}, 0.51 \mathrm{~cm}, 0.56 \mathrm{~cm}$ and $0.54 \mathrm{~cm}$, respectively. Compared with the SPC method, the RMSE of CSM was $0.05 \mathrm{~cm}$.

According to the results, the stem diameters retrieved by the CYF and CF methods are the closest to the field measurement, followed by those of the CLF, CSM, SPC and SP methods. The CLF and CSM methods have similar performance, and the CYF and CF methods have better robustness than the other methods. The equivalency of stem diameter measurement between the diameter tape and the caliper is acceptable for forestry inventories. The stem point preprocessing step is a very important for stem diameter retrieval, and defining the goals and enriching the content of this step (such as stem crosssection completeness checking, stem cross-section validity verification and outlier removal) improves the accuracy of stem diameter retrieval, especially for sample plot scanning.

Author Contributions: Conceptualization, software and writing-original draft preparation, L.Y.; data processing, software, validation and investigation, J.W. and M.L.; investigation and data processing, X.L.; investigation, writing-review and editing, Y.P.; writing—review and editing, X.S. All authors have read and agreed to the published version of the manuscript.

Funding: This research was funded in part by the National Natural Science Foundation of China (No.31872704 31800539 and 61761003), the National Key Research and Development Program (No.2017YFD0600404), the Foundation for Distinguished Young Talents in Higher Education of Henan (No.2020GGJS157), the Public Welfare Project of Ningbo Science and Technology Bureau (No.2019C10084), the Innovation Team Support Plan of University Science and Technology of Henan Province (No.19IRTSTHN014), the Key Scientic Research Project in Colleges and Universities of Henan Province (No.21A520039), and the Nanhu Scholars Program for Young Scholars of XYNU.

Institutional Review Board Statement: Not applicable.

Informed Consent Statement: Not applicable.

Data Availability Statement: Not applicable.

Conflicts of Interest: The authors declare no conflict of interest.

\section{References}

1. Pueschel, P. The influence of scanner parameters on the extraction of tree metrics from FARO Photon 120 terrestrial laser scans. ISPRS J. Photogramm. Remote Sens. 2013, 78, 58-68. [CrossRef]

2. Liang, X.; Hyyppä, J.; Kaartinen, H.; Lehtomäki, M.; Pyörälä, J.; Pfeifer, N.; Holopainen, M.; Brolly, G.; Francesco, P.; Hackenberg, J.; et al. International benchmarking of terrestrial laser scanning approaches for forest inventories. ISPRS J. Photogramm. Remote Sens. 2018, 144, 137-179. [CrossRef]

3. Cabo, C.; Ordóñez, C.; López-Sánchez, C.A.; Armesto, J. Automatic dendrometry: Tree detection, tree height and diameter estimation using terrestrial laser scanning. Int. J. Appl. Earth Obs. Geoinf. 2018, 69, 164-174. [CrossRef]

4. Ritter, T.; Schwarz, M.; Tockner, A.; Leisch, F.; Nothdurft, A. Automatic Mapping of Forest Stands Based on Three-Dimensional Point Clouds Derived from Terrestrial Laser-Scanning. Forests 2017, 8, 265. [CrossRef]

5. Xia, S.; Wang, C.; Pan, F.; Xi, X.; Zeng, H.; Liu, H. Detecting Stems in Dense and Homogeneous Forest Using Single-Scan TLS. Forests 2015, 6, 3923-3945. [CrossRef] 
6. Ravaglia, J.; Fournier, R.A.; Bac, A.; Véga, C.; Côté, J.F.; Piboule, A.; Rémillard, U. Comparison of Three Algorithms to Estimate Tree Stem Diameter from Terrestrial Laser Scanner Data. Forests 2019, 10, 599. [CrossRef]

7. Pitkänen, T.P.; Raumonen, P.; Kangas, A. Measuring stem diameters with TLS in boreal forests by complementary fitting procedure. ISPRS J. Photogramm. Remote Sens. 2019, 147, 294-306. [CrossRef]

8. You, L.; Tang, S.; Song, X.; Lei, Y.; Zang, H.; Lou, M.; Zhuang, C. Precise Measurement of Stem Diameter by Simulating the Path of Diameter Tape from Terrestrial Laser Scanning Data. Remote Sens. 2016, 8, 717. [CrossRef]

9. Srinivasan, S.; Popescu, S.C.; Eriksson, M.; Sheridan, R.D.; Ku, N.W. Terrestrial Laser Scanning as an Effective Tool to Retrieve Tree Level Height, Crown Width, and Stem Diameter. Remote Sens. 2015, 7, 1877-1896. [CrossRef]

10. Kankare, V.; Liang, X.; Vastaranta, M.; Yu, X.; Holopainen, M.; Hyyppä, J. Diameter distribution estimation with laser scanning based multisource single tree inventory. ISPRS J. Photogramm. Remote Sens. 2015, 108, 161-171. [CrossRef]

11. Luoma, V.; Saarinen, N.; Wulder, M.A.; White, J.C.; Vastaranta, M.; Holopainen, M.; Hyyppä, J. Assessing Precision in Conventional Field Measurements of Individual Tree Attributes. Forests 2017, 8, 38. [CrossRef]

12. Hunčaga, M.; Chudá, J.; Tomaštík, J.; Slámová, M.; Koreň, M.; Chudý, F. The Comparison of Stem Curve Accuracy Determined from Point Clouds Acquired by Different Terrestrial Remote Sensing Methods. Remote Sens. 2020, 12, 2739. [CrossRef]

13. Liang, X.; Kankare, V.; Yu, X.; Hyyppä, J.; Holopainen, M. Automated Stem Curve Measurement Using Terrestrial Laser Scanning. IEEE Trans. Geosci. Remote Sens. 2014, 52, 1739-1748. [CrossRef]

14. Ninni Saarinen, V.K.; Vastaranta, M.; Luoma, V.; Pyörälä, J.; Tanhuanpää, T.; Liang, X.; Kaartinen, H.; Kukko, A.; Jaakkola, A.; Yu, X.; et al. Feasibility of Terrestrial laser scanning for collecting stem volume information from single trees. ISPRS J. Photogramm. Remote Sens. 2017, 123, 140-158. [CrossRef]

15. Nölke, N.; Fehrmann, L.; I Nengah, S.; Tiryana, T.; Seidel, D.; Kleinn, C. On the geometry and allometry of big-buttressed trees-a challenge for forest monitoring: New insights from 3D-modeling with terrestrial laser scanning. iForest-Biogeosci. For. 2015, 8, 574. [CrossRef]

16. Liang, X.; Kankare, V.; Hyyppä, J.; Wang, Y.; Kukko, A.; Haggrën, H.; Yu, X.; Kaartinen, H.; Jaakkola, A.; Guan, F.; et al. Terrestrial laser scanning in forest inventories. ISPRS J. Photogramm. Remote Sens. 2016, 115, 63-77. [CrossRef]

17. Kachamba, D.J.; Eid, T. Total tree, merchantable stem and branch volume models for miombo woodlands of Malawi. South. For. J. For. Sci. 2016, 78, 41-51. [CrossRef]

18. You, L.; Guo, J.; Pang, Y.; Tang, S.; Song, X.; Zhang, X. 3D stem model construction with geometry consistency using terrestrial laser scanning data. Int. J. Remote Sens. 2021, 42, 714-737. [CrossRef]

19. Koreň, M.; Hunčaga, M.; Chudá, J.; Mokroš, M.; Surový, P. The Influence of Cross-Section Thickness on Diameter at Breast Height Estimation from Point Cloud. ISPRS Int. J. Geo-Inf. 2020, 9, 495. [CrossRef]

20. Wang, D.; Kankare, V.; Puttonen, E.; Hollaus, M.; Pfeifer, N. Reconstructing stem cross section shapes from terrestrial laser scanning. IEEE Geosci. Remote Sens. Lett. 2017, 14, 272-276. [CrossRef]

21. Stovall, A.E.; Vorster, A.G.; Anderson, R.S.; Evangelista, P.H.; Shugart, H.H. Non-destructive aboveground biomass estimation of coniferous trees using terrestrial LiDAR. Remote Sens. Environ. 2017, 200, 31-42. [CrossRef]

22. Liu, G.; Wang, J.; Dong, P.; Chen, Y.; Liu, Z. Estimating Individual Tree Height and Diameter at Breast Height (DBH) from Terrestrial Laser Scanning (TLS) Data at Plot Level. Forests 2018, 9, 398. [CrossRef]

23. Olofsson, K.; Holmgren, J.; Olsson, H. Tree Stem and Height Measurements using Terrestrial Laser Scanning and the RANSAC Algorithm. Remote Sens. 2014, 6, 4323-4344. [CrossRef]

24. Gao, S.; Zhang, H.; Liu, M. Morphological Parameters Extraction of Tree Branches Based on Point Cloud. J. Northeast. For. Univ. 2014, 42, 109-114.

25. Van Laar, A.; Akca, A. Forest Mensuration; Springer Science \& Business Media: Berlin/Heidelberg, Germany, $2007 ;$ Volume 13.

26. Binot, J.M.; Pothier, D.; Lebel, J. Comparison of relative accuracy and time requirement between the caliper, the diameter tape and an electronic tree measuring fork. For. Chron. 1995, 71. [CrossRef]

27. Tang, S. The theoretical comparison of measuring diameter using caliper and stem diameter tape. For. Resour. Manag. 1977, 23-26.

28. FARO Technologies, I. FARO Focus3D X330. 2013. Available online: https://www.faro.com/en-gb/news/the-new-faro-laser-s canner-focus3d-x-330-the-perfect-instrument-for-3d-documentation-and-land-surveying-2/ (accessed on 26 April 2021).

29. Liu, L.; Pan, g.Y.; Li, Z. Individual Tree DBH and Height Estimation Using Terrestrial Laser Scanning (TLS) in A Subtropical Forest. Sci. Silvae Sin. 2016, 52, 26-37. [CrossRef]

30. Eberly, D. Geometric Tools Engine 5.1. 2020. Available online: https://www.geometrictools.com/GTE/Mathematics/ApprCyl inder3.h (accessed on 26 April 2021).

31. Piegl, L.; Tiller, W. The NURBS Book; Springer: Berlin/Heidelberg, Germany, 1997.

32. You, L.; Feng, Y.; Guo, J.; Ye, J.; Tang, S.; Song, X. Construction of Closed, Global Convexity and G² Continutiy Curve. J. Comput. Aided Des. Comput. Graph. 2017, 30, 309-315. [CrossRef]

33. Pfeifer, N.; Winterhalder, D. Modelling of tree cross sections from terrestrial laser scanning data with free-form curves. Int. Arch. Photogramm. Remote Sens. Spat. Inf. Sci. 2004, 36, W2.

34. Ye, W.; Qian, C.; Tang, J.; Liu, H.; Fan, X.; Liang, X.; Zhang, H. Improved 3D Stem Mapping Method and Elliptic Hypothesis-Based DBH Estimation from Terrestrial Laser Scanning Data. Remote Sens. 2020, 12, 352. [CrossRef]

35. Nurunnabi, A.; Sadahiro, Y.; Lindenbergh, R.; Belton, D. Robust cylinder fitting in laser scanning point cloud data. Measurement 2019, 138, 632-651. [CrossRef] 